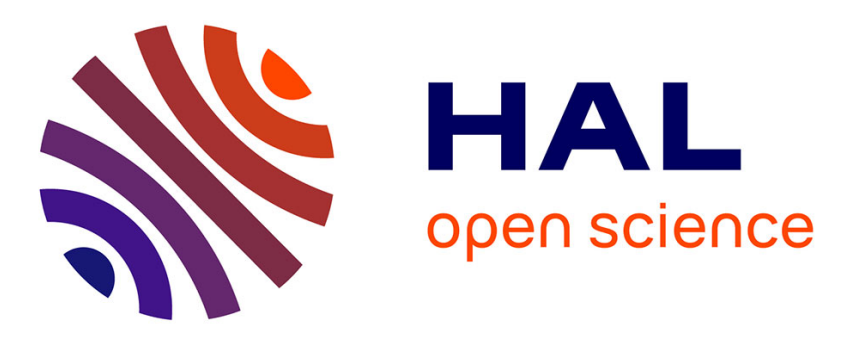

\title{
Thermal Event Recognition Applied to Tokamak Protection during Plasma Operation
}

Vincent Martin, François Bremond, Jean-Marcel Travere, Victor Moncada, Gwenaël Dunand

\section{- To cite this version:}

Vincent Martin, François Bremond, Jean-Marcel Travere, Victor Moncada, Gwenaël Dunand. Thermal Event Recognition Applied to Tokamak Protection during Plasma Operation. International Instrumentation and Measurement Technology Conference, May 2009, Singapore, Singapore. pp.16901694, 10.1109/IMTC.2009.5168728 . inria-00499628

\section{HAL Id: inria-00499628 \\ https://hal.inria.fr/inria-00499628}

Submitted on 11 Jul 2010

HAL is a multi-disciplinary open access archive for the deposit and dissemination of scientific research documents, whether they are published or not. The documents may come from teaching and research institutions in France or abroad, or from public or private research centers.
L'archive ouverte pluridisciplinaire HAL, est destinée au dépôt et à la diffusion de documents scientifiques de niveau recherche, publiés ou non, émanant des établissements d'enseignement et de recherche français ou étrangers, des laboratoires publics ou privés. 


\section{Thermal Event Recognition Applied to Tokamak Protection during Plasma Operation}

\author{
Vincent Martin, François Brémond \\ INRIA Sophia Antipolis Méditerrannée \\ 2004 route des Lucioles, BP93 \\ F-06902 Sophia Antipolis, France. \\ Email: \{vmartin,fbremond\}@ sophia.inria.fr
}

\author{
Jean-Marcel Travere, Victor Moncada, Gwenaël Dunand \\ CEA, IRFM \\ F-13108 Saint Paul-lez-Durance, France. \\ Email: $\{$ jean-marcel.travere,victor.moncada, gwenael.dunand $\} @$ cea.fr
}

\begin{abstract}
Magnetic confinement fusion reactors are complex devices where a large amount of energy is required to make the fusion reactions happen. In such experimental conditions, the Plasma Facing Components (PFC) are subjected to high heat fluxes. In current tokamaks like Tore Supra, infrared thermographic diagnostics based on image analysis and feedback control are used to measure and monitor the heating of the PFC during plasma operation. The system consists in detecting high increase of the IR luminance signal beyond fixed temperature thresholds for a set of predefined Regions of Interest (ROI). Consequently, this system neither takes into account the thermal objects outside of the ROI, nor the geometric information of the detected thermal object. In this paper, we propose a new visionbased approach for the automatic detection of thermal events. This approach is composed of three main tasks: thermal object detection (1), classification (2), and thermal event recognition (3). We present results of our approach for the recognition of one critical thermal event and compare it with the previous system.
\end{abstract}

\section{INTRODUCTION}

Magnetic confinement fusion is a more and more active field of research since nuclear fusion is considered as a promising approach for alternative energy production. One issue to reach a sufficient power balance in the future devices like ITER is to rely on long plasma pulses with high auxiliary heating. The goal is to inject most of the available power while ensuring the Plasma Facing Components (PFC) safety by limiting the power load just below their operational limits. To measure and monitor the heating of the PFC during plasma operation, the most efficient way is to collect surface temperature information by using a network of infrared video cameras. Then a feedback control based on infrared image analysis is used to control the heat loads in real-time. Such a system has been implemented at Tore Supra where infrared cameras are monitoring in realtime the five heating antennas. The image analysis part of the Tore Supra's feedback control consists in detecting high increase of the IR luminance signal beyond fixed temperature thresholds for a set of predefined Regions of Interest (ROI) (see figure 1). It has been successfully applied to the detection of some thermal events [1], as electric arcing. These arcs are caused by a potential difference between the two lateral shields of a heating antenna and can lead to the destruction of sensitive parts like the copper grills (see figure 2). However, this system has several drawbacks and limitations:

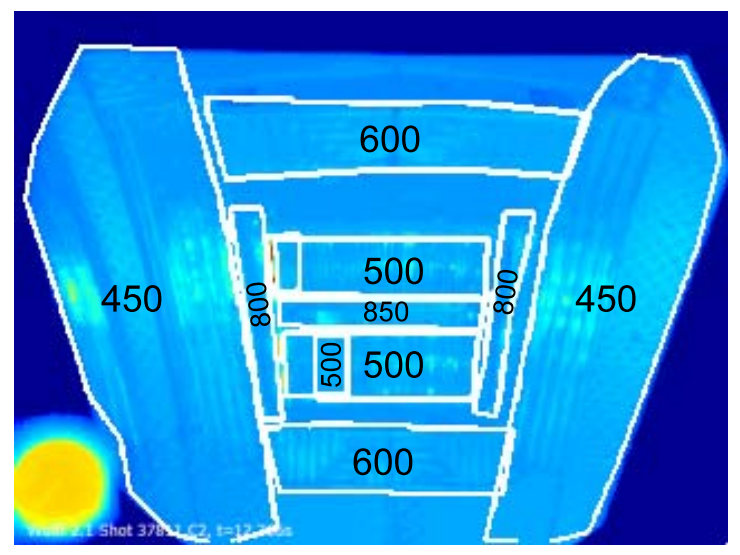

Fig. 1. ROI drawn by the user for the monitoring and the feedback control to prevent from PFC overheating. Temperature thresholds (in ${ }^{\circ} \mathrm{C}$ ) are indicated for each ROI.
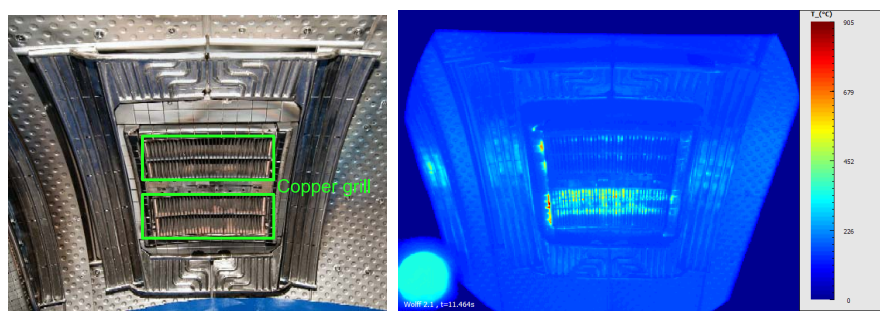

Fig. 2. A heating antenna with the its infrared image during heat load. Electric arcing is visible on the bottom part of the infrared image.

1) working with temperature values makes the strong assumption that the infrared digital sensors are always well calibrated and the optical transmission factors are wellknown,

2) it requires an operator for both editing the ROI each time the PFC configuration changes, manually tune the temperature thresholds,

3) thermal events occuring outside of the ROI cannot be detected,

4) the sensitivity to false detection is high: the presence of only one noisy or dead pixel in a ROI with a temperature higher than the detection threshold is enough to raise a 
false alarm,

5) different thermal events will not be discriminated if they occur in the same ROI.

In summary, a need exists in both improvement of the video processing and better understanding and interpretation of infrared images as, for instance, an accurate identification of new heating zones (hot spots). In this paper, we propose a new approach for the automatic detection of Thermal Events (TE) inspired from a video understanding framework [2] widely used in video surveillance applications. The goal is to separate the domain expert knowledge on event modeling from the image processing tasks dedicated to the extraction of low level features from visual data. The major advantage of such a framework compared to the current system is that the a priori knowledge of one event is not inside the image but in the event model description.

\section{RECOGNITION OF THERMAL EVENTS}

The global dynamic of the infrared image sequences can be classified into two states: steady and transient. During steady state, the average level of the pixel values does not change or very slowly. Transient states occur when the power injection is increased or modulated. In this case, the pixel variations can be sudden. We call a thermal object a local region in the image which has a high contrast with its neighbourhood. A description of identified critical areas of potential overheat and their related heat source can be found in [3]. Two kinds of thermal objects can be detected in the infrared images: normal and abnormal. Usually normal thermal object dynamics evolve with respect to the heat load whereas abnormal ones have a thermal intensity which can unexpectedly increase, especially during steady states.

Four representatives thermographic behaviours of specific patterns during a pulse are shown in figure 3 and are described below:

(1) background pixels: the temporal evolution is practically null,

(2) reflection area from metallic component (normal TE),

(3) hot spot: this is a normal thermal event caused by the interaction of fast electrons with the PFC (guard limiter). The temperature increases linearly in the time,

(4) electric arcing: strong temperature peaks during the steady state (abnormal TE).

The major difficulty is to efficiently detect such thermal events in a dynamic experimental context with a low false alarm rate. To this end, we follow a process chain as illustrated in figure 4 where each of the three main vision tasks is guided by the domain expert knowledge.

\section{A. a priori knowledge formalization}

More precisely, we use a formalized visual description of the thermal events to detect. This description relies on a visual concept ontology composed of generic spatiotemporal attributes such as geometric and temporal cues [4]. The main advantage in using such a symbolic description is its reusability for other thermal events to recognize. Indeed, visual

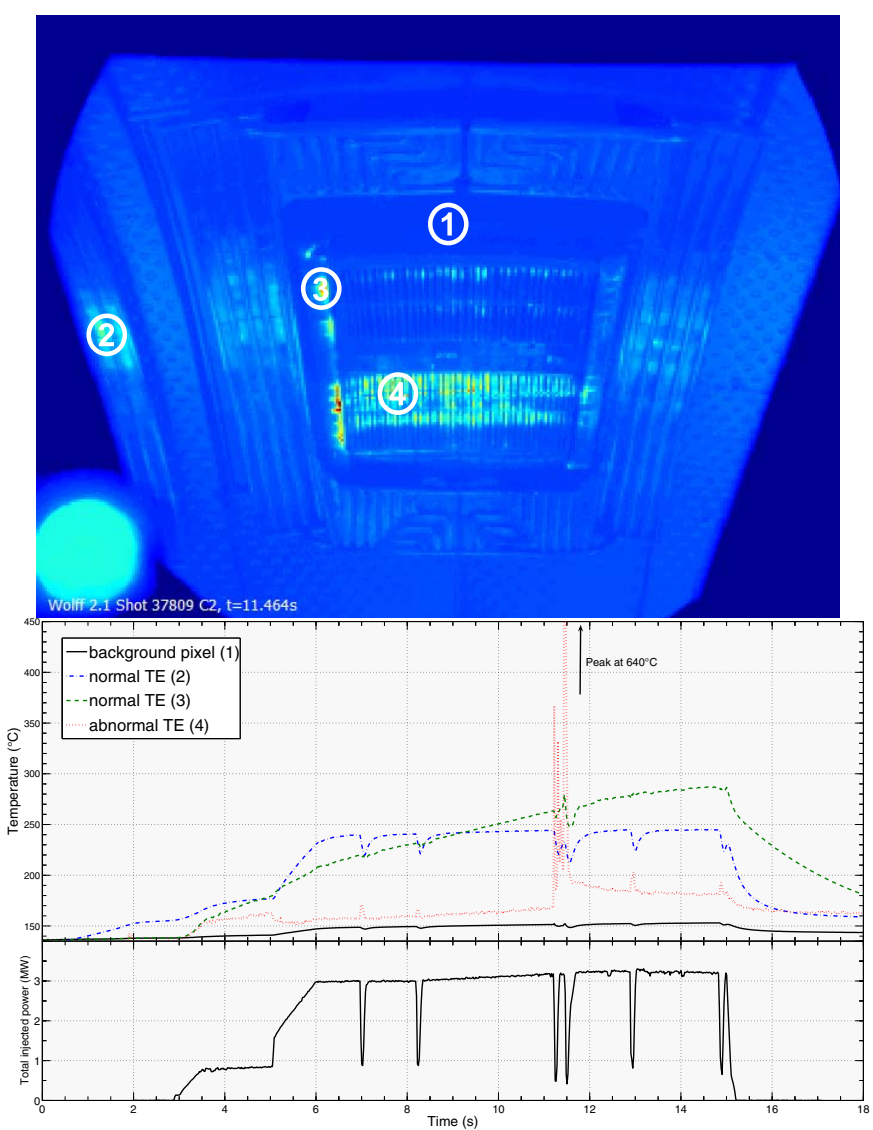

Fig. 3. Temporal evolution of 5 local areas (picked from the infrared frame on top) and of the heating power injection (bottom plot).

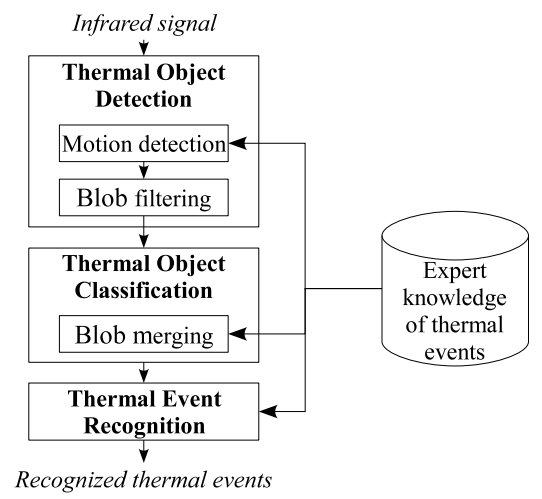

Fig. 4. Process chain of the proposed approach.

concepts are an intermediate level that helps mapping lowlevel numerical values to a domain class description. For the arcing event, the description used is described in figure 5.

The local contrast of a region $r$ is defined as the ratio $\frac{\left|\mu_{r}-\mu_{n r}\right|}{\left(\mu_{r}+\mu_{n r}\right)}$ where $\mu_{r}$ is the mean value of the pixels in region $r$ and $\mu_{n r}$ the mean value of the pixels surrounding the region $r$. The $\Delta T$ velocity is the positive variation of the temperature $\Delta T=T_{i}-T_{i-1}$ between the time interval $\Delta t=t_{i}-t_{i-1}$. 
Domain Class Electrical arcing SuperClass Abnormal Thermal Event Spatial Attributes

\begin{tabular}{|c|c|c|}
\hline Length & [ 50 & 110 \\
\hline Height & {$[7$} & 20 \\
\hline Elongation & {$[2.5$} & 16 \\
\hline Area & {$[120$} & 2200 \\
\hline \multicolumn{3}{|l|}{ ral Attributes } \\
\hline Duration & [ 1 & 8 \\
\hline $\begin{array}{l}\Delta T \text { velocity } \\
\text { rature Attributes }\end{array}$ & {$[154$} & 347 \\
\hline Local contrast & {$[1.3 e-4$} & 0.17 \\
\hline Entropy & {$[3.8$} & 9.02 \\
\hline
\end{tabular}

Fig. 5. High-level description of an electric arc. Spatial attributes have been translated from physical dimensions to pixel values. Temporal attributes have been translated from seconds to frames. Ranges values corresponds to observed min and max.

\section{B. Thermal object detection}

Motion is a particularly important cue for object detection in image sequences. Indeed, a moving object is often referred as an object of interest and can then be classified as foreground. The simplest approach to detect moving pixel in image sequences consist in subtracting the current frame from a reference frame. Since background is rarely stationary, the key issue for any background subtraction algorithm is to efficiently model the background and update it according to the pixel variations. Many approaches have been proposed to background modeling based on unimodal distribution, mixture of gaussians or compressed models (see [5] for an overview). Here the method of Butler et al. [6] is used. The basic idea of this method is to represent each pixel in the frame by a group of clusters. The clusters are sorted in order of the likelihood that they model the background and are adapted to deal with background changes. The algorithm depends on three main free parameters: the number of clusters, the adaptation rate, and the foreground classification threshold. The adaptation rate is directly linked up to the duration and the thermal evolution of the thermal event to detect. A small rate will be adapted to the detection of slow thermal events whereas a high rate will be adapted to the detection of rapid thermal events such as electric arcing. Therefore the setting of this parameter depends directly on the a priori knowledge given by the experts. In the same way, the foreground classification threshold can be connected to the temperature attributes. Basically, the parameter is set according to the local contrast attribute of the thermal event. Concerning the number of clusters, we observed only small detection differences while varying the value (between 2 and 5), so we decided to set it to 3 in order to maintain a good trade-off between sensitivity and frame rate.

Electrical arcs are oriented horizontally (in the video camera referential) with a strong infrared signature at the copper grill level. Nevertheless, it is very rare to observe the entire pattern in the infrared images. Most of the time, only a collection of pattern subparts are visible and detected in the form of several blobs by the segmentation algorithm. This is why a specific merging process is necessary to reconstruct the whole arcing pattern.

\section{Thermal object classification}

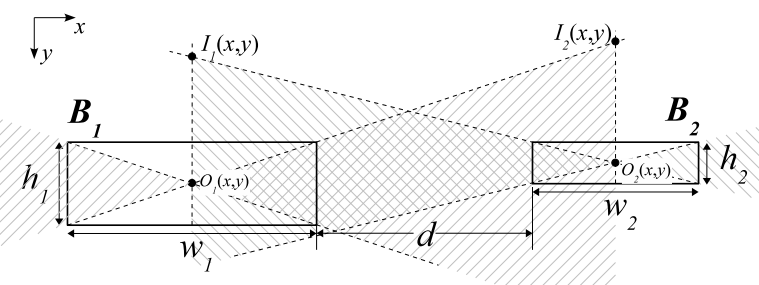

Fig. 6. Blobs features $(w, h, o)$ and inter-blob features $(d, I)$ used by the merging criteria.

According to the symbols used in figure 6, we have defined a blob merging criterion as follows:

$$
H V C=\left\{\left|y_{O_{i}}-y_{O_{j}}\right|<\left|y_{I_{i}}-y_{O_{j}}\right|, \forall i, j=1,2, i \neq j\right\}
$$

This criterion favors the merge of blobs having the same orientation (here horizontal for the arcs) and capable of seeing each other in this orientation. Two blobs $B_{1}$ and $B_{2}$ are defined as visible for each other if the center of $B_{1}$ (resp. $B_{2}$ ) is inside the hatched zone formed by $B_{2}$ (resp. $B_{1}$ ) as seen in figure 6 . The visibility is proportional to the inverse of the distance, to the sizes, and to the horizontal elongations of the two blobs (parameters $d, w$, and $h$ ). This criterion prevents from merging distant small blobs. Then, a new bounding box including the two merged blobs is computed and the merging process is repeated until no more merges are possible.

\section{Thermal event recognition}

Once each blob has been classified, the last task is to recognize the event patterns from the detected thermal objects. To this end, we also rely on the a priori knowledge of figure 5 . For the specific case of electric arcing, we use three criteria for the event recognition: the size of the pattern, its shape (i.e. horizontal rectangle), and its duration. This step is essential for the precise spatial localization of the event patterns.

\section{EXPERIMENTAL RESULTS}

To assess the performance of our system, we have compared the results of the arcing event detection with ground truth data obtained from manual annotations of a pulse dataset. The pulse dataset used for this evaluation is composed of 50 infrared films taken from two heating antennas (the two lower hybrid current drive launchers). The videos are acquired at 50 frames per seconds. The frame size is $320 \times 240$ pixels. The spatial resolution is around $4 \times 4 \mathrm{~mm}^{2}$. A pulse duration is between 5 and 62 seconds. This corresponds to a total plasma time of 1496 seconds. The frame rate of the $\mathrm{C}++$ software implementation on $2 \times 2.33 \mathrm{GHz} \mathrm{PC}$ is about 23 frames per second. A total of 183 arcing events have been annotated. Counting results are reported in table I.

False negative results can be explained by the lack of precision of some annotations (see figure 7) and by the limits of the merging criteria. False positive results are due to 

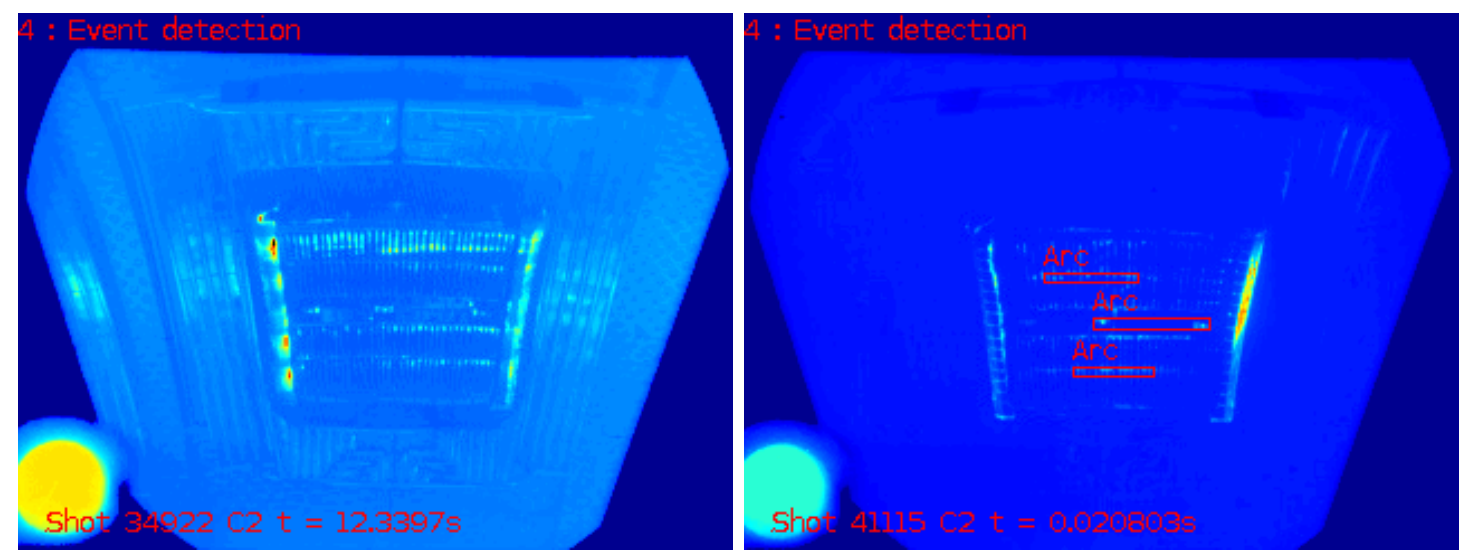

Fig. 7. Two examples of typical bad detection. On the left image: the electric arcs on the top of the grill have not been detected. On the right image: false positive example during plasma priming.

\begin{tabular}{|c|c|c|c|c|c|}
\hline \multirow[t]{2}{*}{ Antenna } & \multirow{2}{*}{$\begin{array}{l}\text { no. of } \\
\text { pulses }\end{array}$} & \multirow[t]{2}{*}{ GT } & \multicolumn{3}{|c|}{ no. of detected arcing events } \\
\hline & & & $\mathrm{TP}$ & FN & FP \\
\hline $\mathrm{C} 2$ & 28 & 138 & 135 & 3 & 4 \\
\hline $\mathrm{C} 3$ & 22 & 45 & 41 & 4 & 3 \\
\hline $\mathrm{C} 2+\mathrm{C} 3$ & 50 & 183 & 176 & 7 & 7 \\
\hline
\end{tabular}

TABLE I

DETECTION RESULTS FOR THE ARCING EVENT WITH GROUND TRUTH (GT), TRUE POSITIVE (TP), FALSE NEGATIVE (FN), AND FALSE POSITIVE (FP) COUNTS.

detection errors during the transient states. In particular, false alarms often occurs during the plasma priming (see figure 7). A simple triggering of the detection algorithm during the transient states should be enough to prevent from such false detections. Figure 8 presents visual results at each step of the system and for three representative cases.

\section{CONCLUSION AND FUTURE WORK}

We have proposed a vision-based system for the automatic recognition of thermal events in infrared images of PFC in Tokamaks. The proposed system overcomes the weaknesses of the current thermal event detection system used at Tore Supra by more precise localization and characterization of each TE while using a minimum of explicit knowledge (visual descriptions with corresponding attributes) and basic image processings. If this system has been applied to only one critical TE, it can be easily extended to the recognition of others TE. To this end, we may take advantage of fusion massive databases [7] for the automatic selection and learning of TE attributes by means of data mining techniques. Another way to improve the system performance is to take into account the uncertainty on the algorithm outputs in the system decisions.

The ultimate goal of this system is to improve the reliability of the current real-time control acting on the heating sources. To reach real-time constraints, we plan to implement the thermal object detection algorithm on a FPGA . Finally, such a system could be a solution for the ITER project where wide angle video cameras are planned for the PFC monitoring.

\section{ACKNOWLEDGMENT}

This work, supported by the European Communities under the contract of Association between EURATOM and CEA, was carried out within the framework of the European Fusion Development Agreement. The views and opinions expressed herein do not necessarily reflect those of the European Commission.

\section{REFERENCES}

[1] J.M. Travere, et al., "Image analysis of infrared quantitative data applied to tokamak survey and safety: present and future," IEEE International Symposium on Intelligent Signal Processing, WISP, pp. 1-5, Oct. 2007.

[2] F. Brémond, M. Thonnat, and M. Zuniga, "Video understanding framework for automatic behavior recognition," Journal on Behavior Research Methods, vol. 3, no. 38, pp. 416-426, 2006.

[3] M. Chatelier on behalf of Equipe Tore Supra, "Integration of high power, long pulse operation in tore supra in preparation for iter," Nuclear Fusion, vol. 47, no. 10, pp. S579-S589, 2007.

[4] N. Maillot and M. Thonnat, "Ontology based complex object recognition,' Image Vision Comput., vol. 26, no. 1, pp. 102-113, 2008.

[5] R. Radke, S. Andra, O. Al-Kofahi, and B. Roysam, "Image change detection algorithms: a systematic survey," IEEE Transactions on Image Processing,, vol. 14, no. 3, pp. 294-307, March 2005.

[6] D. E. Butler, J. V. Michael Bove, and S. Sridharan, "Real-time adaptive foreground/background segmentation," EURASIP J. Appl. Signal Process., vol. 2005, no. 1, pp. 2292-2304, 2005.

[7] J. Vega and et al., "Recent results on structural pattern recognition for fusion massive databases," IEEE International Symposium on Intelligent Signal Processing, WISP, pp. 1-6, Oct. 2007 

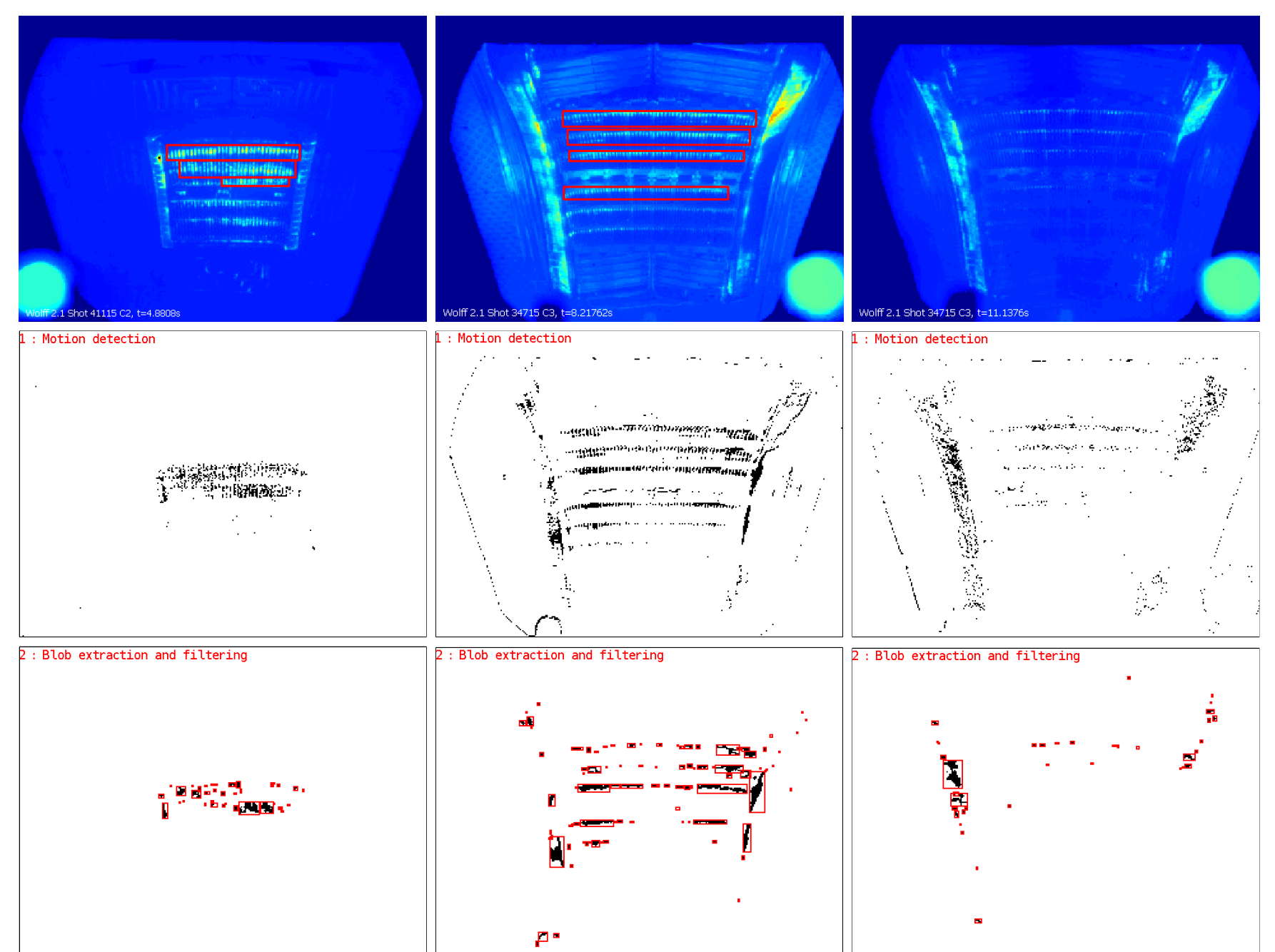

2 : Blob extraction and filtering
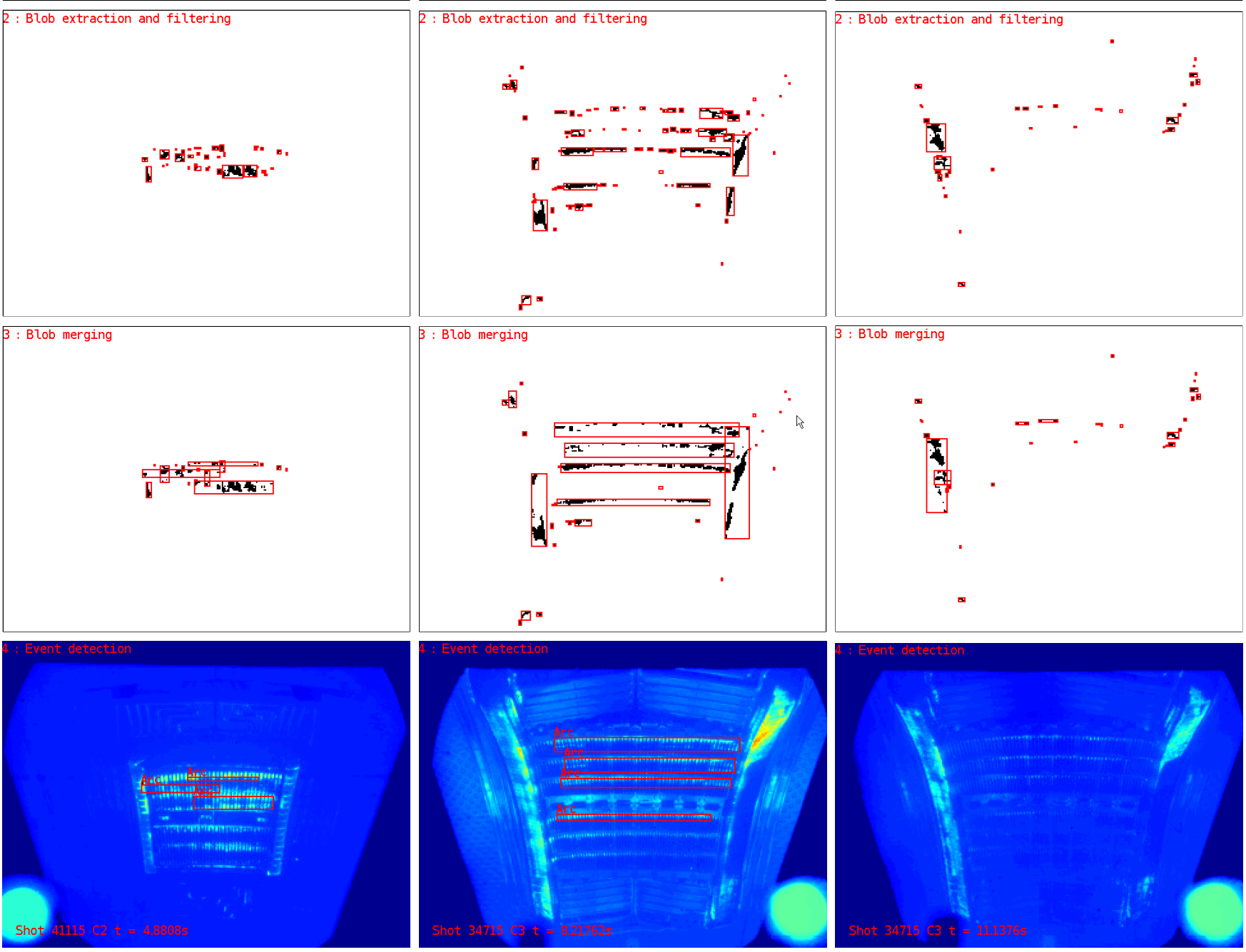

Fig. 8. Ground truth with corresponding bounding box superimposed on original images (row 1), system outputs after motion detection, foreground pixels are in black (row 2), median filtering and blob extraction (row 3), blob merging (row 4), and arcing event recognition with corresponding bounding box superimposed on original images (row 5). 